MATHEMATICS OF COMPUTATION

Volume 65, Number 216

October 1996, Pages 1463-1475

\title{
THE ORDER OF CONVERGENCE OF EIGENFREQUENCIES IN FINITE ELEMENT APPROXIMATIONS OF FLUID-STRUCTURE INTERACTION PROBLEMS
}

\author{
RODOLFO RODRÍGUEZ AND JORGE E. SOLOMIN
}

\begin{abstract}
In this paper we prove a double order for the convergence of eigenfrequencies in fluid-structure vibration problems. We improve estimates given recently for compressible and incompressible fluids. To do this, we extend classical results on finite element spectral approximation to nonconforming methods for noncompact operators.
\end{abstract}

\section{INTRODUCTION}

This paper deals with the approximation of eigenfrequencies in vibration problems for coupled systems with fluid-structure interaction. Such problems have been considered in several recent papers. A survey of current results and references can be found in $[12]$.

In particular, we consider the interior elastoacoustics problem consisting of determining the vibration modes of an elastic solid containing a fluid. Different formulations have been proposed to solve this problem (see $[6,11,14]$ ). A pure displacement formulation yields a simple eigenvalue problem; however, spurious modes arise when standard finite element methods are applied to it (see [11]). Recently, several methods to avoid these spurious modes have been proposed (see $[2,3,8]$ ).

In [2] a finite element method consisting of Raviart-Thomas elements for the displacements of the fluid and standard Courant elements for those of the solid is introduced for the case of a compressible fluid. A weak coupling between both type of elements along the interface is imposed. In [3] this method has been adapted to deal with incompressible fluids. Both procedures yield nonconforming methods to approximate the eigenvalue problem for the noncompact operators present in the formulation.

It is well known, in the case of conforming approximations of compact operators, that the order of convergence for the eigenvalues doubles that for the eigenfunctions (see, for instance, [1]). In [10] the same result is proved to be valid even for noncompact operators under reasonable assumptions. A similar analysis is contained in [13] for nonconforming approximations of compact operators. None of these theories cover the situations considered in [2] and [3].

Received by the editor January 30, 1995.

1991 Mathematics Subject Classification. Primary 65N25, 65N30; Secondary 70J30, 73K70, $76 \mathrm{Q} 05$.

Key words and phrases. Fluid-structure, eigenvalue problems.

Partially supported by Consejo Nacional de Investigaciones Científicas y Técnicas, Argentina.

(C)1996 American Mathematical Society 
In [5] and [4] efficient numerical results are reported in the cases of compressible and incompressible fluids, respectively. In both cases, the experiments seem to show an improved order of convergence for the eigenvalues. The aim of this paper is to show that this order effectively doubles that for the eigenfunctions. Toward this end, a suitable modification of the theory in [10] is introduced to cover the nonconforming case. By so doing, consistency terms arise in the estimates. These terms are proved to be of the appropriate order in the present cases.

In $\S 2$ we give a general result valid for nonconforming approximations of noncompact operators. In $\S 3$ and $\S 4$ we apply this result to the cases of a compressible and an incompressible fluid, respectively.

\section{Nonconforming approximation of the EigenVAlues OF A NONCOMPACT OPERATOR}

Let $\mathbf{X}$ be a complex Hilbert space with norm $\|\cdot\|$. Let $\mathbf{V}$ be a closed subspace of $\mathbf{X}$ and $\left\{\mathbf{V}_{h}\right\}$ a family of finite-dimensional subspaces of $\mathbf{X}$. Let $a$ and $b$ be two continuous sesquilinear forms on $\mathbf{X} ; a$ is assumed to be coercive. Let $A$ and $A_{h}: \mathbf{X} \longrightarrow \mathbf{X}$ be the linear operators defined for each $\mathbf{x} \in \mathbf{X}$ by

$$
\begin{aligned}
A \mathbf{x} \in \mathbf{V}: & a(A \mathbf{x}, \mathbf{y})=b(\mathbf{x}, \mathbf{y}), \quad \forall \mathbf{y} \in \mathbf{V}, \\
A_{h} \mathbf{x} \in \mathbf{V}_{h}: & a\left(A_{h} \mathbf{x}, \mathbf{y}\right)=b(\mathbf{x}, \mathbf{y}), \quad \forall \mathbf{y} \in \mathbf{V}_{h} .
\end{aligned}
$$

Because of the Lax-Milgram lemma, the operators $A$ and $A_{h}$ are bounded uniformly in $h$.

If $\mathbf{V} \neq \mathbf{X}$, then $\sigma(A)=\sigma(A \mid \mathbf{V}) \cup\{0\}$, where $\sigma(\cdot)$ denotes the spectrum of an operator. Let $\lambda \in \mathbf{C}$ be an isolated eigenvalue of $\left.A\right|_{\mathbf{V}}$ of finite algebraic multiplicity $m$; since $a$ is coercive, $\lambda \neq 0$. Therefore, $\lambda$ is also an isolated eigenvalue of $A$. Let $D \subset \mathbf{C}$ be a closed circle centered at $\lambda$ such that $0 \notin D$ and $D \cap \sigma(A)=\{\lambda\}$. Let $\lambda_{1 h}, \ldots, \lambda_{m(h) h}$ be the eigenvalues of $A_{h}$ contained in $D$ (repeated according to their algebraic multiplicities).

We assume the following two approximation properties to be satisfied:

P1: There holds

$$
\lim _{h \rightarrow 0}\left\|\left(A-A_{h}\right) \mid \mathbf{v}_{h}\right\|=0
$$

P2: For each eigenfunction $\mathbf{x}$ of $A$ associated with $\lambda$, there holds

$$
\lim _{h \rightarrow 0}\left(\inf _{\mathbf{x}_{h} \in \mathbf{V}_{h}}\left\|\mathbf{x}-\mathbf{x}_{h}\right\|\right)=0 .
$$

Under these assumptions, it was proved in [9] that for $h$ small enough one has $m(h)=m$ and that $\lim _{h \rightarrow 0} \lambda_{i h}=\lambda$ for $i=1, \ldots, m$. In [10] estimates of $\left|\lambda_{i}-\lambda_{i h}\right|$ are given in the conforming case (i.e., when $\mathbf{V}_{h} \subset \mathbf{V}$ ).

In [13] the same problem is analyzed but assuming

P3: There holds

$$
\lim _{h \rightarrow 0}\left\|A-A_{h}\right\|=0
$$

instead of P1 and P2. However, P3 implies that $A$ is compact, which is not the case in the fluid-structure vibration problems considered in the following sections. Furthermore, the results in [13] giving an improved order of convergence for the eigenvalues rely on a similar property holding in weaker norms for suitable restrictions of $A-A_{h}$. This does not seem to be the case in the applications under consideration since incomplete polynomials of degree one are used for the fluid. 
The results in [9] could be extended to the nonconforming case (i.e., $\mathbf{V}_{h} \not \subset \mathbf{V}$ ). However for the estimators therein to be useful, the eigenfunctions of the adjoint operator $A^{*}$ with respect to $a$ need to be smooth. To avoid this drawback, instead of the adjoints $A^{*}$ and $A_{h}^{*}$, we consider as in [13] the bounded operators $A_{*}$ and $A_{* h}: \mathbf{X} \longrightarrow \mathbf{X}$ defined by

$$
\begin{aligned}
A_{*} \mathbf{x} \in \mathbf{V}: & a\left(\mathbf{y}, A_{*} \mathbf{x}\right)=b(\mathbf{y}, \mathbf{x}), \quad \forall \mathbf{y} \in \mathbf{V}, \\
A_{* h} \mathbf{x} \in \mathbf{V}_{h}: & a\left(\mathbf{y}, A_{* h} \mathbf{x}\right)=b(\mathbf{y}, \mathbf{x}), \quad \forall \mathbf{y} \in \mathbf{V}_{h} .
\end{aligned}
$$

Then $\bar{\lambda}$ is an eigenvalue of $A_{*}$ with the same multiplicity $m$ as that of $\lambda$ (see [13]). Analogously, for $h$ small enough, $\bar{\lambda}_{1 h}, \ldots, \bar{\lambda}_{m h}$ are the eigenvalues of $A_{* h}$ belonging to $\bar{D}:=\{\bar{\mu} \in \mathbf{C}: \mu \in D\}$.

We assume for $A_{*}$ and $A_{* h}$ approximation properties analogous to P1 and P2, namely,

$\mathbf{P} 1_{*}$ : There holds

$$
\lim _{h \rightarrow 0}\left\|\left.\left(A_{*}-A_{* h}\right)\right|_{\mathbf{v}_{h}}\right\|=0 ;
$$

$\mathbf{P 2} 2_{*}$ : For each eigenfunction $\mathbf{x}$ of $A_{*}$ associated with $\bar{\lambda}$, there holds

$$
\lim _{h \rightarrow 0}\left(\inf _{\mathbf{x}_{h} \in \mathbf{V}_{h}}\left\|\mathbf{x}-\mathbf{x}_{h}\right\|\right)=0 .
$$

Let $\Pi_{h}$ and $\Pi_{* h}: \mathbf{X} \longrightarrow \mathbf{X}$ be the projectors with range $\mathbf{V}_{h}$ defined by $a\left(\mathbf{x}-\Pi_{h} \mathbf{x}, \mathbf{y}\right)=0, \forall \mathbf{y} \in \mathbf{V}_{h}$, and $a\left(\mathbf{y}, \mathbf{x}-\Pi_{* h} \mathbf{x}\right)=0, \forall \mathbf{y} \in \mathbf{V}_{h}$, respectively. Since $a$ is continuous and coercive, $\Pi_{h}$ and $\Pi_{* h}$ are bounded uniformly in $h$. Let $B_{h}:=A_{h} \Pi_{h}: \mathbf{X} \longrightarrow \mathbf{X}$. Notice that $\sigma\left(A_{h}\right)=\sigma\left(B_{h}\right)$ and that, for any nonzero eigenvalue, the corresponding invariant subspaces coincide. Let $B_{* h}:=A_{* h} \Pi_{* h}: \mathbf{X} \longrightarrow \mathbf{X}$.

Let $E: \mathbf{X} \longrightarrow \mathbf{X}$ be the spectral projector of $A$ relative to the isolated eigenvalue $\lambda$. Let $F_{h}: \mathbf{X} \longrightarrow \mathbf{X}$ be the spectral projector of $B_{h}$ relative to its eigenvalues $\lambda_{1 h}, \ldots, \lambda_{m h}$. It can be proved as in Lemma 1 of [10] that there exists $h_{0}>0$ such that $\left\|\left(z-B_{h}\right)^{-1}\right\|$ is bounded for $h \leq h_{0}$ and $z \in \partial D$; consequently, for $h$ small enough, the spectral projectors $F_{h}$ are bounded uniformly in $h$. Let $E_{*}$ and $F_{* h}$ be the spectral projector of $A_{*}$ and $B_{* h}$ relative to $\bar{\lambda}$ and $\bar{\lambda}_{1 h}, \ldots, \bar{\lambda}_{m h}$, respectively.

We remark that $B_{* h}$ turns out to be the actual adjoint of $B_{h}$ with respect to $a$. In fact, for all $\mathbf{x}$ and $\mathbf{y} \in \mathbf{X}$,

$$
a\left(B_{h} \mathbf{x}, \mathbf{y}\right)=a\left(A_{h} \Pi_{h} \mathbf{x}, \mathbf{y}\right)=a\left(A_{h} \Pi_{h} \mathbf{x}, \Pi_{* h} \mathbf{y}\right)=b\left(\Pi_{h} \mathbf{x}, \Pi_{* h} \mathbf{y}\right)
$$

and, analogously, $a\left(\mathbf{x}, B_{* h} \mathbf{y}\right)=b\left(\Pi_{h} \mathbf{x}, \Pi_{* h} \mathbf{y}\right)$. Therefore, the spectral projector $F_{* h}$ is also the adjoint of $F_{h}$.

For $\mathbf{Y}$ and $\mathbf{Z}$ closed subspaces of $\mathbf{X}$, let

$$
\delta(\mathbf{Y}, \mathbf{Z}):=\sup _{\substack{\mathbf{y} \in \mathbf{Y} \\\|\mathbf{y}\|=1}}\left(\inf _{\mathbf{z} \in \mathbf{Z}}\|\mathbf{y}-\mathbf{z}\|\right)
$$

and

$$
\widehat{\delta}(\mathbf{Y}, \mathbf{Z}):=\max \{\delta(\mathbf{Y}, \mathbf{Z}), \delta(\mathbf{Z}, \mathbf{Y})\} .
$$

Let $\gamma_{h}:=\delta\left(E(\mathbf{X}), \mathbf{V}_{h}\right)$ and $\gamma_{* h}:=\delta\left(E_{*}(\mathbf{X}), \mathbf{V}_{h}\right)$. Because of $\mathbf{P 2}$ and $\mathbf{P 2} 2_{*}$, we have $\gamma_{h} \rightarrow 0$ and $\gamma_{* h} \rightarrow 0$ as $h \rightarrow 0$. Since $a$ is coercive and continuous, there holds $\left\|\left.\left(I-\Pi_{h}\right)\right|_{E(\mathbf{X})}\right\| \leq \boldsymbol{C} \gamma_{h}$ and $\left\|\left.\left(I-\Pi_{* h}\right)\right|_{E_{*}(\mathbf{X})}\right\| \leq \boldsymbol{C} \gamma_{* h}$. From now on, $\boldsymbol{C}$ 
denotes a generic constant not necessarily the same at each occurrence but always independent of $h$.

For $h$ sufficiently small, we have the following results:

Lemma 2.1. There holds

$$
\begin{aligned}
\left\|\left.\left(E-F_{h}\right)\right|_{E(\mathbf{X})}\right\| & \leq \boldsymbol{C}\left\|\left.\left(A-B_{h}\right)\right|_{E(\mathbf{X})}\right\|, \\
\left\|\left.\left(E_{*}-F_{* h}\right)\right|_{E_{*}(\mathbf{X})}\right\| & \leq \boldsymbol{C}\left\|\left.\left(A_{*}-B_{* h}\right)\right|_{E_{*}(\mathbf{X})}\right\| .
\end{aligned}
$$

Proof. The proof is identical to that of Lemma 3 in [10].

Let $\delta_{h}:=\gamma_{h}+\left\|\left.\left(A-A_{h}\right)\right|_{\mathbf{V}_{h}}\right\|$ and $\delta_{* h}:=\gamma_{* h}+\left\|\left(A_{*}-A_{* h}\right) \mid \mathbf{v}_{h}\right\|$. Because of P1 and $\mathbf{P 2}$ (resp. $\mathbf{P} \mathbf{1}_{*}$ and $\mathbf{P} \mathbf{2}_{*}$ ) we have $\delta_{h} \rightarrow 0$ and $\delta_{* h} \rightarrow 0$ as $h \rightarrow 0$.

Lemma 2.2. There holds

$$
\begin{aligned}
\left\|\left.\left(A-B_{h}\right)\right|_{E(\mathbf{X})}\right\| & \leq \boldsymbol{C} \delta_{h}, \\
\left\|\left.\left(A_{*}-B_{* h}\right)\right|_{E_{*}(\mathbf{X})}\right\| & \leq \boldsymbol{C} \delta_{* h} .
\end{aligned}
$$

Proof. Let $\mathbf{x} \in E(\mathbf{X})$ with $\|\mathbf{x}\|=1$; we have

$$
\begin{aligned}
\left\|\left(A-B_{h}\right) \mathbf{x}\right\| & \leq\left\|A\left(I-\Pi_{h}\right) \mathbf{x}\right\|+\left\|\left(A-A_{h}\right) \Pi_{h} \mathbf{x}\right\| \\
& \leq\|A\|\left\|\left(I-\Pi_{h}\right) \mathbf{x}\right\|+\left\|\left(A-A_{h}\right) \mid \mathbf{v}_{h}\right\|\left\|\Pi_{h} \mathbf{x}\right\| \\
& \leq \boldsymbol{C}\left(\gamma_{h}+\left\|\left(A-A_{h}\right) \mid \mathbf{v}_{h}\right\|\right)=\boldsymbol{C} \delta_{h},
\end{aligned}
$$

and an analogous proof is valid for the second estimate of the theorem.

Let $\Lambda_{h}:=\left.F_{h}\right|_{E(\mathbf{X})}: E(\mathbf{X}) \longrightarrow F_{h}(\mathbf{X})$.

Lemma 2.3. The operator $\Lambda_{h}$ is bijective and $\left\|\Lambda_{h}^{-1}\right\|$ is bounded in $h$.

Proof. See the proof of Theorem 1 in [10].

Theorem 2.1. There holds

$$
\widehat{\delta}\left(F_{h}(\mathbf{X}), E(\mathbf{X})\right) \leq \boldsymbol{C} \delta_{h} .
$$

Proof. The proof is identical to that of Theorem 1 in [10].

Let $\hat{A}:=\left.A\right|_{E(\mathbf{X})}: E(\mathbf{X}) \longrightarrow E(\mathbf{X})$ and $\hat{B}_{h}:=\Lambda_{h}^{-1} B_{h} \Lambda_{h}: E(\mathbf{X}) \longrightarrow E(\mathbf{X})$. The operator $\hat{A}$ has a unique eigenvalue $\lambda$ of algebraic multiplicity $m$; the operator $\hat{B}_{h}$ has the eigenvalues $\bar{\lambda}_{1 h}, \ldots, \bar{\lambda}_{m h}$.

Consider the following consistency terms:

$$
\begin{gathered}
M_{h}=\sup _{\substack{\mathbf{x} \in E(\mathbf{X}) \\
\|\mathbf{x}\|=1}} \sup _{\substack{\mathbf{y} \in E_{*}(\mathbf{X}) \\
\|\mathbf{y}\|=1}}\left[a\left(A \mathbf{x}, \Pi_{* h} \mathbf{y}\right)-b\left(\mathbf{x}, \Pi_{* h} \mathbf{y}\right)\right], \\
M_{* h}=\sup _{\substack{\mathbf{x} \in E(\mathbf{X}) \\
\|\mathbf{x}\|=1}} \sup _{\substack{\mathbf{y} \in E_{*}(\mathbf{X}) \\
\|\mathbf{y}\|=1}}\left[a\left(\Pi_{h} \mathbf{x}, A_{*} \mathbf{y}\right)-b\left(\Pi_{h} \mathbf{x}, \mathbf{y}\right)\right] .
\end{gathered}
$$

It is clear that $M_{h}=M_{* h}=0$ for conforming methods. In general, we have the following result, which can be seen to be an extension to the nonconforming case of Theorem 2 in [10].

Theorem 2.2. There holds

$$
\left\|\hat{A}-\hat{B}_{h}\right\| \leq \boldsymbol{C}\left(\delta_{h} \delta_{* h}+M_{h}+M_{* h}\right) .
$$


Proof. We easily see that

$$
\begin{aligned}
\left\|\hat{A}-\hat{B}_{h}\right\| & =\sup _{\substack{\mathbf{x} \in E(\mathbf{X}) \\
\|\mathbf{x}\|=1}}\left\|\left(\hat{A}-\hat{B}_{h}\right) \mathbf{x}\right\| \\
& \leq \boldsymbol{C} \sup _{\substack{\mathbf{x} \in E(\mathbf{X}) \\
\|\mathbf{x}\|=1}} \sup _{\substack{\mathbf{y} \in \mathbf{V} \\
\|\mathbf{y}\|=1}} a\left(\left(\hat{A}-\hat{B}_{h}\right) \mathbf{x}, \mathbf{y}\right) \\
& =\mathbf{C} \sup _{\substack{\mathbf{x} \in E(\mathbf{X}) \\
\|\mathbf{x}\|=1}} \sup _{\mathbf{y} \in E_{*}(\mathbf{X})} a((\hat{A} \|=1
\end{aligned}
$$

By using that $\left.\left(\Lambda_{h}^{-1} F_{h}-I\right) A\right|_{E(\mathbf{X})}=0$ and that $B_{h}$ commutes with its spectral projector $F_{h}$, it is straightforward to prove that

$$
\hat{A}-\hat{B}_{h}=\left.\left(A-B_{h}\right)\right|_{E(\mathbf{X})}+\left.\left(\Lambda_{h}^{-1} F_{h}-I\right)\left(A-B_{h}\right)\right|_{E(\mathbf{X})} .
$$

Let $\mathbf{x} \in E(\mathbf{X})$ and $\mathbf{y} \in E_{*}(\mathbf{X})$ with $\|\mathbf{x}\|=\|\mathbf{y}\|=1$. Since $F_{h}\left(\Lambda_{h}^{-1} F_{h}-I\right)=0$ and $F_{* h}$ is the adjoint of $F_{h}$ with respect to $a$, we have

$$
\begin{aligned}
\left|a\left(\left(\Lambda_{h}^{-1} F_{h}-I\right)\left(A-B_{h}\right) \mathbf{x}, \mathbf{y}\right)\right| \\
\quad=\left|a\left(\left(\Lambda_{h}^{-1} F_{h}-I\right)\left(A-B_{h}\right) \mathbf{x},\left(I-F_{* h}\right) \mathbf{y}\right)\right| \\
\quad \leq\|a\|\left\|\Lambda_{h}^{-1} F_{h}-I\right\|\left\|\left.\left(A-B_{h}\right)\right|_{E(\mathbf{X})}\right\|\left\|\left.\left(I-F_{* h}\right)\right|_{E_{* h}(\mathbf{X})}\right\| \\
\quad \leq \boldsymbol{C} \delta_{h} \delta_{* h},
\end{aligned}
$$

where we have used Lemmas 2.1, 2.2 and 2.3 and the fact that $F_{h}$ is bounded independently of $h$.

On the other hand,

$$
a\left(\left(A-B_{h}\right) \mathbf{x}, \mathbf{y}\right)=a\left(\left(A-B_{h}\right) \mathbf{x}, \Pi_{* h} \mathbf{y}\right)+a\left(\left(A-B_{h}\right) \mathbf{x},\left(I-\Pi_{* h}\right) \mathbf{y}\right) .
$$

By using Lemma 2.2 we may bound the second term in the right-hand side of (2.8):

$$
\begin{aligned}
\left|a\left(\left(A-B_{h}\right) \mathbf{x},\left(I-\Pi_{* h}\right) \mathbf{y}\right)\right| & \leq\|a\|\left\|\left.\left(A-B_{h}\right)\right|_{E(\mathbf{X})}\right\|\left\|\left.\left(I-\Pi_{* h}\right)\right|_{E_{* h}(\mathbf{X})}\right\| \\
& \leq \boldsymbol{C} \delta_{h} \gamma_{* h},
\end{aligned}
$$

whereas for the first term we have

$$
a\left(\left(A-B_{h}\right) \mathbf{x}, \Pi_{* h} \mathbf{y}\right)=a\left(\left(A-A_{h}\right) \mathbf{x}, \Pi_{* h} \mathbf{y}\right)+a\left(\left(A_{h}-B_{h}\right) \mathbf{x}, \Pi_{* h} \mathbf{y}\right)
$$

Now,

$$
\left|a\left(\left(A-A_{h}\right) \mathbf{x}, \Pi_{* h} \mathbf{y}\right)\right|=\left|a\left(A \mathbf{x}, \Pi_{* h} \mathbf{y}\right)-b\left(\mathbf{x}, \Pi_{* h} \mathbf{y}\right)\right| \leq M_{h}
$$

and

$$
\begin{aligned}
a\left(\left(A_{h}-B_{h}\right) \mathbf{x}, \Pi_{* h} \mathbf{y}\right) & =a\left(A_{h}\left(I-\Pi_{h}\right) \mathbf{x}, \Pi_{* h} \mathbf{y}\right)=b\left(\left(I-\Pi_{h}\right) \mathbf{x}, \Pi_{* h} \mathbf{y}\right) \\
& =b\left(\left(I-\Pi_{h}\right) \mathbf{x}, \mathbf{y}\right)-b\left(\left(I-\Pi_{h}\right) \mathbf{x},\left(I-\Pi_{* h}\right) \mathbf{y}\right) .
\end{aligned}
$$

The second term in the right-hand side of (2.12) can be easily bounded by

$$
\begin{aligned}
\left|b\left(\left(I-\Pi_{h}\right) \mathbf{x},\left(I-\Pi_{* h}\right) \mathbf{y}\right)\right| & \leq\|b\|\left\|\left.\left(I-\Pi_{h}\right)\right|_{E(\mathbf{X})}\right\|\left\|\left.\left(I-\Pi_{* h}\right)\right|_{E_{* h}(\mathbf{X})}\right\| \\
& \leq \boldsymbol{C} \gamma_{h} \gamma_{* h},
\end{aligned}
$$


and for the first term we have

$$
\begin{aligned}
b\left(\left(I-\Pi_{h}\right) \mathbf{x}, \mathbf{y}\right) & =a\left(\mathbf{x}, A_{*} \mathbf{y}\right)-b\left(\Pi_{h} \mathbf{x}, \mathbf{y}\right) \\
& =a\left(\left(I-\Pi_{h}\right) \mathbf{x}, A_{*} \mathbf{y}\right)+\left[a\left(\Pi_{h} \mathbf{x}, A_{*} y\right)-b\left(\Pi_{h} \mathbf{x}, \mathbf{y}\right)\right] .
\end{aligned}
$$

Finally, for the first term on the right of (2.14) we have that

$$
\begin{aligned}
\left|a\left(\left(I-\Pi_{h}\right) \mathbf{x}, A_{*} \mathbf{y}\right)\right| & =\left|a\left(\left(I-\Pi_{h}\right) \mathbf{x},\left(I-\Pi_{* h}\right) A_{*} \mathbf{y}\right)\right| \\
& \leq\|a\|\left\|\left.\left(I-\Pi_{h}\right)\right|_{E(\mathbf{x})}\right\|\left\|\left.\left(I-\Pi_{* h}\right)\right|_{E_{* h}(\mathbf{X})}\right\|\left\|A_{*}\right\| \leq \boldsymbol{C} \gamma_{h} \gamma_{* h}
\end{aligned}
$$

whereas for the second term we have

$$
\left|a\left(\Pi_{h} \mathbf{x}, A_{*} y\right)-b\left(\Pi_{h} \mathbf{x}, \mathbf{y}\right)\right| \leq M_{* h} .
$$

Now the theorem is a consequence of formulae (2.5) to (2.16).

By using the previous theorem, we deduce the following result about the approximation of the eigenvalue $\lambda$ :

Theorem 2.3. We have

$$
\left|\lambda-\frac{1}{m} \sum_{i=1}^{m} \lambda_{i h}\right| \leq \boldsymbol{C}\left(\delta_{h} \delta_{* h}+M_{h}+M_{* h}\right)
$$

and

$$
\max _{i=1, \ldots, m}\left|\lambda-\lambda_{i h}\right| \leq \boldsymbol{C}\left(\delta_{h} \delta_{* h}+M_{h}+M_{* h}\right)^{1 / \alpha},
$$

where $\alpha$ is the ascent of the eigenvalue $\lambda$ of $\hat{A}$ (i.e., $\alpha$ is the least positive integer such that $\left.\operatorname{Ker}\left((\lambda-\hat{A})^{\alpha}\right)=\operatorname{Ker}\left((\lambda-\hat{A})^{\alpha+1}\right)\right)$.

Proof. (2.17) is a direct consequence of Theorem 2.2 and the continuity of the trace for finite-dimensional operators. (2.18) is an application of well-known results for matrices (see, for instance, [16]).

We remark that if $\left.A\right|_{E(\mathbf{X})}$ is diagonalizable, then $\alpha=1$ and so we have estimates of the same order for the convergence of all the approximate eigenvalues $\lambda_{1 h}, \ldots, \lambda_{m h}$. This is the case in the applications considered in the following sections, where $\left.A\right|_{\mathbf{V}}: \mathbf{V} \longrightarrow \mathbf{V}$ is selfadjoint with respect to $a$.

\section{Approximation of Eigenfrequencies in A Fluid-STRUCTURE PRoblem}

We consider the problem of determining the vibration modes of an ideal (inviscid) barotropic fluid contained in a linear elastic structure. We take as a model problem the case of a vessel completely filled by the fluid. We restrict ourselves to the $2 \mathrm{D}$ case and assume polygonal boundaries and interfaces. This problem has been analyzed in [2], where a nonoptimal rate of convergence for the eigenfrequencies has been proved.

Let $\Omega_{\mathrm{F}}$ and $\Omega_{\mathrm{S}}$ be the domains occupied by the fluid and the solid, respectively, as in Figure 1. We assume $\Omega_{\mathrm{F}}$ to be simply connected but not necessarily convex. By $\Gamma_{\mathrm{I}}$ we denote the interface between the solid and the fluid, and by $\boldsymbol{\nu}$ its unit normal vector pointing outward from $\Omega_{\mathrm{F}}$. The exterior boundary of the solid is the union of $\Gamma_{\mathrm{D}}$ and $\Gamma_{\mathrm{N}}$ : the structure is fixed along $\Gamma_{\mathrm{D}}$ and free of stress along $\Gamma_{\mathrm{N}}$; let $\boldsymbol{\eta}$ denote the unit outward normal vector along $\Gamma_{\mathrm{N}}$. 


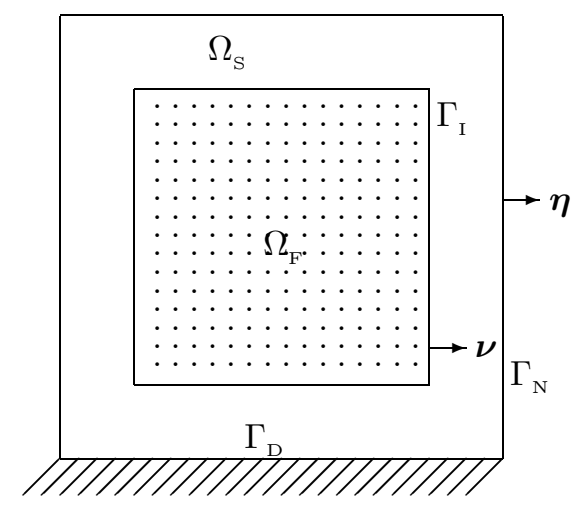

Figure 1. Fluid and solid domains

Henceforth, we use the standard notation for Sobolev spaces, norms and seminorms.

Let $\mathbf{X}:=H\left(\operatorname{div}, \Omega_{\mathrm{F}}\right) \times\left[H_{\Gamma_{\mathrm{D}}}^{1}\left(\Omega_{\mathrm{S}}\right)\right]^{2}\left(H_{\Gamma_{\mathrm{D}}}^{1}\left(\Omega_{\mathrm{S}}\right)\right.$ being the subspace of functions in $H^{1}\left(\Omega_{\mathrm{S}}\right)$ vanishing on $\left.\Gamma_{\mathrm{D}}\right)$ endowed with the $H\left(\operatorname{div}, \Omega_{\mathrm{F}}\right) \times\left[H^{1}\left(\Omega_{\mathrm{S}}\right)\right]^{2}$ norm, which we denote by $\|(\mathbf{u}, \mathbf{v})\|$. Let $\mathbf{V}$ be the closed subspace of $\mathbf{X}$ defined by $\mathbf{V}:=\left\{(\mathbf{u}, \mathbf{v}) \in \mathbf{X}: \mathbf{u} \cdot \boldsymbol{\nu}=\mathbf{v} \cdot \boldsymbol{\nu}\right.$ on $\left.\Gamma_{\mathrm{I}}\right\}$. Let $a_{0}$ and $b$ be the continuous, symmetric and positive bilinear forms on $\mathbf{X}$ given by

$$
\begin{aligned}
a_{0}((\mathbf{u}, \mathbf{v}),(\boldsymbol{\phi}, \boldsymbol{\psi})) & :=\int_{\Omega_{\mathrm{F}}} \rho_{\mathrm{F}} c^{2} \operatorname{div} \mathbf{u} \operatorname{div} \boldsymbol{\phi}+\int_{\Omega_{\mathrm{S}}} \boldsymbol{\sigma}(\mathbf{v}): \varepsilon(\boldsymbol{\psi}), \\
b((\mathbf{u}, \mathbf{v}),(\boldsymbol{\phi}, \boldsymbol{\psi})) & :=\int_{\Omega_{\mathrm{F}}} \rho_{\mathrm{F}} \mathbf{u} \cdot \boldsymbol{\phi}+\int_{\Omega_{\mathrm{S}}} \rho_{\mathrm{S}} \mathbf{v} \cdot \boldsymbol{\psi},
\end{aligned}
$$

where $\boldsymbol{\sigma}(\mathbf{v}): \varepsilon(\psi)$ denotes the tensorial product between the strain tensor $\varepsilon_{i j}(\boldsymbol{\psi}):=$ $\frac{1}{2}\left(\frac{\partial \psi_{i}}{\partial x_{j}}+\frac{\partial \psi_{j}}{\partial x_{i}}\right)$ and the stress tensor $\sigma_{i j}(\mathbf{v}):=\lambda_{\mathrm{S}} \sum_{k=1}^{2} \varepsilon_{k k}(\mathbf{v}) \delta_{i j}+2 \mu_{\mathrm{S}} \varepsilon_{i j}(\mathbf{v})\left(\lambda_{\mathrm{S}}\right.$ and $\mu_{\mathrm{S}}$ being the Lamé coefficients of the structure); $\rho_{\mathrm{S}}$ and $\rho_{\mathrm{F}}$ are the densities of the solid and the fluid, respectively, and $c$ is the acoustic speed in the fluid.

The classical acoustic approximation for the small-amplitude motions yields the following eigenvalue problem for the vibration modes of the coupled system (see $[2])$ :

$\mathbf{V P}_{0}$ : Find $\lambda \in \mathbf{R}$ and $(\mathbf{u}, \mathbf{v}) \in \mathbf{V},(\mathbf{u}, \mathbf{v}) \neq(\mathbf{0}, \mathbf{0})$, such that

$$
a_{0}((\mathbf{u}, \mathbf{v}),(\boldsymbol{\phi}, \boldsymbol{\psi}))=\lambda b((\mathbf{u}, \mathbf{v}),(\boldsymbol{\phi}, \boldsymbol{\psi})), \quad \forall(\boldsymbol{\phi}, \boldsymbol{\psi}) \in \mathbf{V} .
$$

Here, $\omega=\sqrt{\lambda}$ is the frequency of the eigenmode, and $\mathbf{u}$ and $\mathbf{v}$ are the displacements in the fluid and in the solid, respectively.

There exist eigenmodes of problem $\mathbf{V P}_{0}$ which do not induce vibrations into the solid. They are pure rotational motions of the fluid and correspond to the eigenvalue $\lambda=0$. It is proved in [2] that the corresponding eigenspace is $\mathbf{K}:=$ $\left\{(\operatorname{curl} \xi, \mathbf{0}), \xi \in H_{0}^{1}\left(\Omega_{\mathrm{F}}\right)\right\}$. Therefore, the bilinear form $a_{0}$ is not coercive. However, $a:=a_{0}+b$ is coercive and can be used instead. The eigenvalue problem associated with $a$ is:

VP: Find $\lambda \in \mathbf{R}$ and $(\mathbf{u}, \mathbf{v}) \in \mathbf{V},(\mathbf{u}, \mathbf{v}) \neq(\mathbf{0}, \mathbf{0})$, such that

$$
a((\mathbf{u}, \mathbf{v}),(\boldsymbol{\phi}, \boldsymbol{\psi}))=\lambda b((\mathbf{u}, \mathbf{v}),(\boldsymbol{\phi}, \boldsymbol{\psi})), \quad \forall(\boldsymbol{\phi}, \boldsymbol{\psi}) \in \mathbf{V} .
$$


As in the previous section, we consider the bounded linear operator $A: \mathbf{X} \longrightarrow \mathbf{X}$ defined by $A(\mathbf{u}, \mathbf{v}) \in \mathbf{V}$ and

$$
a(A(\mathbf{u}, \mathbf{v}),(\boldsymbol{\phi}, \boldsymbol{\psi}))=b((\mathbf{u}, \mathbf{v}),(\boldsymbol{\phi}, \boldsymbol{\psi})), \quad \forall(\boldsymbol{\phi}, \boldsymbol{\psi}) \in \mathbf{V} .
$$

Since $a$ and $b$ are symmetric, $\left.A\right|_{\mathbf{V}}$ is selfadjoint with respect to $a$. Clearly, $(\lambda,(\mathbf{u}, \mathbf{v}))$ is an eigenpair of $\left.A\right|_{\mathbf{v}}$ if and only if $\left(\frac{1}{\lambda},(\mathbf{u}, \mathbf{v})\right)$ is a solution of VP which, in turn, is equivalent to $\left(\frac{1}{\lambda}-1,(\mathbf{u}, \mathbf{v})\right)$ being a solution of $\mathbf{V} \mathbf{P}_{0}$.

The operator $\left.A\right|_{\mathbf{V}}$ is not compact; in fact, $\lambda=1$ is an eigenvalue of $\left.A\right|_{\mathbf{V}}$ with infinite-dimensional eigenspace $\mathbf{K}$. Nevertheless, $A_{\mathbf{K}^{\perp}}$ is compact. This is used in [2] to show that the spectrum of $\left.A\right|_{\mathbf{V}}$ consists of the eigenvalue $\lambda=1$ and a sequence of finite-multiplicity eigenvalues $\left\{\lambda_{n}: n \in \mathbf{N}\right\} \subset(0,1)$ converging to 0 . Each eigenvector $\left(\mathbf{u}_{n}, \mathbf{v}_{n}\right)$ associated with $\lambda_{n} \in(0,1)$ satisfies curl $\mathbf{u}_{n}=0$. Furthermore, additional smoothness can be proved for these eigenmodes. In particular, the following a priori estimate will be used below to estimate the consistency terms.

Theorem 3.1. Let $(\mathbf{u}, \mathbf{v})$ be an eigenfunction of $A$ with corresponding eigenvalue $\lambda \in(0,1)$. Then $\mathbf{u} \in\left[H^{\alpha}\left(\Omega_{\mathrm{F}}\right)\right]^{2}, \mathbf{v} \in\left[H^{1+\beta}\left(\Omega_{\mathrm{S}}\right)\right]^{2}$, $\operatorname{div} \mathbf{u} \in H^{1+\alpha}\left(\Omega_{\mathrm{F}}\right)$ and

$$
\|\mathbf{u}\|_{\left[H^{\alpha}\left(\Omega_{\mathrm{F}}\right)\right]^{2}}+\|\mathbf{v}\|_{\left[H^{1+\beta}\left(\Omega_{\mathrm{S}}\right)\right]^{2}}+\|\operatorname{div} \mathbf{u}\|_{H^{1+\alpha}\left(\Omega_{\mathrm{F}}\right)} \leq \boldsymbol{C}\|(\mathbf{u}, \mathbf{v})\|,
$$

with $\alpha \in(1 / 2,1]$ and $\beta \in(0,1]$ and with $\boldsymbol{C}$ a positive constant.

Proof. This is a straightforward extension of the proof of Theorem 2.6 in [2]. As therein, the constants $\alpha$ and $\beta$ only depend on the data of the problem. More precisely, $\alpha=1$ if $\Omega_{\mathrm{F}}$ is convex, and $\alpha=\frac{\pi}{\theta}$, with $\theta$ the largest reentrant corner of $\Omega_{\mathrm{F}}$, otherwise. On the other hand, $\beta \in(0,1]$ depends on the reentrant corners of $\partial \Omega_{\mathrm{S}}$, on the angles between $\Gamma_{\mathrm{N}}$ and $\Gamma_{\mathrm{D}}$ and on the Lamé coefficients $\lambda_{\mathrm{S}}$ and $\mu_{\mathrm{S}}$.

The eigenfunctions in $\mathbf{K}$ are not physically relevant since they induce neither vibrations into the structure nor gradients of pressure into the fluid. However, a suitable numerical approximation should be such that the discrete eigenspace associated with $\lambda=1$ be large enough as to approximate the whole $\mathbf{K}$ as the meshsize goes to zero. Otherwise, spurious modes may appear. This is the case, for instance, when continuous piecewise linear finite elements are used for both, the fluid and the solid (see [11]).

To avoid such spurious modes, the following approach is considered in [2]. Let $\left\{\mathcal{T}_{h}\right\}$ be a family of regular triangulations of $\Omega_{\mathrm{F}} \cup \Omega_{\mathrm{S}}$ such that every triangle is completely contained either in $\Omega_{\mathrm{F}}$ or in $\Omega_{\mathrm{S}}$. For each component of the displacements in the solid, standard piecewise linear finite element spaces are used:

$$
L_{h}\left(\Omega_{\mathrm{S}}\right):=\left\{v \in H^{1}\left(\Omega_{\mathrm{S}}\right):\left.v\right|_{T} \in \mathcal{P}_{1}(T), \forall T \in \mathcal{T}_{h}, T \subset \Omega_{\mathrm{S}}\right\} .
$$

For the displacements in the fluid, the lowest-order Raviart-Thomas spaces [15] are used:

$$
\mathbf{R}_{h}\left(\Omega_{\mathrm{F}}\right):=\left\{\mathbf{u} \in H\left(\operatorname{div}, \Omega_{\mathrm{F}}\right):\left.\mathbf{u}\right|_{T} \in \mathcal{R}_{0}(T), \forall T \in \mathcal{T}_{h}, T \subset \Omega_{\mathrm{F}}\right\}
$$

where

$$
\mathcal{R}_{0}(T):=\left\{\mathbf{u} \in \mathcal{P}_{1}(T)^{2}: \mathbf{u}(x, y)=(a+b x, c+b y), a, b, c \in \mathbf{R}\right\} .
$$

The degrees of freedom in $\mathbf{R}_{h}\left(\Omega_{\mathrm{F}}\right)$ are the (constant) values of the normal component of $\mathbf{u}$ along each edge of the triangulation. The discrete analogue of $\mathbf{X}$ is

$$
\mathbf{X}_{h}:=\left\{(\mathbf{u}, \mathbf{v}) \in \mathbf{R}_{h}\left(\Omega_{\mathrm{F}}\right) \times\left[L_{h}\left(\Omega_{\mathrm{S}}\right)\right]^{2}:\left.\mathbf{v}\right|_{\Gamma_{\mathrm{D}}}=\mathbf{0}\right\} .
$$


The intersections $\mathbf{V} \cap \mathbf{X}_{h}$ are not suitable finite element spaces to approximate V. In fact, any function of these spaces has constant normal components along each segment of $\Gamma_{\mathrm{I}}$ and, hence, only functions in $\mathbf{V}$ with the same property could be well approximated. Instead, we use

$$
\mathbf{V}_{h}:=\left\{(\mathbf{u}, \mathbf{v}) \in \mathbf{X}_{h}: \int_{\ell}(\mathbf{u} \cdot \boldsymbol{\nu}-\mathbf{v} \cdot \boldsymbol{\nu})=0, \forall \ell \text { edge of } \mathcal{T}_{h}, \ell \subset \Gamma_{\mathrm{I}}\right\} .
$$

We remark that for $(\mathbf{u}, \mathbf{v}) \in \mathbf{V}_{h}, \mathbf{u} \cdot \boldsymbol{\nu}$ and $\mathbf{v} \cdot \boldsymbol{\nu}$ coincide at the midpoint of each edge $\ell \subset \Gamma_{\mathrm{I}}$ but, in general, they do not coincide on the whole edge. Hence, $\mathbf{V}_{h} \not \subset \mathbf{V}$; that is, the method turns out to be nonconforming.

As in the previous section, we consider the linear operator $A_{h}: \mathbf{X} \longrightarrow \mathbf{X}$ defined by $A_{h}(\mathbf{u}, \mathbf{v}) \in \mathbf{V}_{h}$ and

$$
a\left(A_{h}(\mathbf{u}, \mathbf{v}),(\boldsymbol{\phi}, \boldsymbol{\psi})\right)=b((\mathbf{u}, \mathbf{v}),(\boldsymbol{\phi}, \boldsymbol{\psi})), \quad \forall(\boldsymbol{\phi}, \boldsymbol{\psi}) \in \mathbf{V}_{h} .
$$

Properties $\mathbf{P 1}$ and $\mathbf{P 2}$ in the previous section have been proved in [2] for this finite element approximation. More precisely:

P1: There exist positive constants $\boldsymbol{C}$ and $h_{0}$ such that, if $h \leq h_{0}$, then

$$
\left\|\left.\left(A-A_{h}\right)\right|_{\mathbf{v}_{h}}\right\| \leq C h^{\gamma} .
$$

P2: For each eigenfunction $(\mathbf{u}, \mathbf{v})$ of $A$ associated with $\lambda \in(0,1)$ with $\|(\mathbf{u}, \mathbf{v})\|=$ 1 , there exist positive constants $C$ and $h_{0}$ such that, if $h \leq h_{0}$, then

$$
\inf _{\left(\mathbf{u}_{h}, \mathbf{v}_{h}\right) \in \mathbf{V}_{h}}\left\|(\mathbf{u}, \mathbf{v})-\left(\mathbf{u}_{h}, \mathbf{v}_{h}\right)\right\| \leq \boldsymbol{C} h^{\gamma} .
$$

In both cases, $\gamma:=\min \{\alpha, \beta\}$ with $\alpha$ and $\beta$ the constants arising in Theorem 3.1. Obviously, properties $\mathbf{P} \mathbf{1}_{*}$ and $\mathbf{P} \mathbf{2}_{*}$ are equally valid. In fact, since $a$ and $b$ are symmetric, then $A_{*}$ and $A_{* h}$, as defined in (2.3) and (2.4), coincide with $A$ and $A_{h}$, respectively.

The spectrum of $A_{h}$ furnishes the approximation of the spectrum of $\left.A\right|_{\mathbf{V}}$ analyzed in [2]. The following results are proved therein. First, the eigenspace associated with $\lambda=1$ is well represented in this discretization:

Theorem 3.2. $\lambda=1$ is an eigenvalue of $A_{h}$ and its eigenspace is $\mathbf{K} \cap \mathbf{V}_{h}$.

Secondly, there are no spurious eigenvalues for $h$ small enough:

Theorem 3.3. Let $J$ be a closed interval such that $J \cap \sigma(A)=\emptyset$. There exists $h_{J}>0$ such that, if $h \leq h_{J}$, then $J \cap \sigma\left(A_{h}\right)=\emptyset$.

Let $\lambda \in(0,1)$ be an eigenvalue of $A$ with multiplicity $m$. As in the previous section, let $E(\mathbf{X})$ denote the eigenspace of $A$ associated with $\lambda$. Since $\mathbf{P 1}$ and P2 are fulfilled, for $h$ small enough there are exactly $m$ eigenvalues of $A_{h}, \lambda_{1 h}, \ldots, \lambda_{m h}$, (repeated according to their multiplicities) converging to $\lambda$. Let $F_{h}(\mathbf{X})$ denote the eigenspace of $A_{h}$ associated with them. Theorem 2.1 can be rewritten in this context:

Theorem 3.4. There exist positive constants $\boldsymbol{C}$ and $h_{0}$ such that, for $h \leq h_{0}$,

$$
\widehat{\delta}\left(E(\mathbf{X}), F_{h}(\mathbf{X})\right) \leq \boldsymbol{C} h^{\gamma},
$$

with $\gamma:=\min \{\alpha, \beta\}$ and $\alpha$ and $\beta$ as in Theorem 3.1.

In order to analyze the convergence of the $\lambda_{i h}$ 's by means of Theorem 2.3, we need to bound the consistency terms. Notice that $M_{h}$ and $M_{* h}$ also coincide because of the symmetry of $a$ and $b$. 
Lemma 3.1. There exist positive constants $\boldsymbol{C}$ and $h_{0}$ such that, for $h \leq h_{0}$,

$$
M_{h} \leq \boldsymbol{C h} h^{1+\gamma} .
$$

Proof. In our case,

$$
M_{h}=\sup _{\substack{(\mathbf{u}, \mathbf{v}) \in E(\mathbf{X}) \\\|(\mathbf{u}, \mathbf{v})\|=1}} \sup _{\substack{\phi, \psi(\psi) \in E(\mathbf{X}) \\\|(\phi, \psi)\|=1}}\left[a\left(A(\mathbf{u}, \mathbf{v}), \Pi_{h}(\boldsymbol{\phi}, \boldsymbol{\psi})\right)-b\left((\mathbf{u}, \mathbf{v}), \Pi_{h}(\boldsymbol{\phi}, \boldsymbol{\psi})\right)\right],
$$

with $\Pi_{h}$ being the projection onto $\mathbf{V}_{h}$ with respect to $a$.

Let $(\mathbf{u}, \mathbf{v}),(\boldsymbol{\phi}, \boldsymbol{\psi}) \in E(\mathbf{X})$ and $\left(\phi_{h}, \boldsymbol{\psi}_{h}\right):=\Pi_{h}((\boldsymbol{\phi}, \boldsymbol{\psi}))$; proceeding as in the proof of Lemma 5.7 in [2], we obtain

$$
a\left(A(\mathbf{u}, \mathbf{v}), \Pi_{h}(\boldsymbol{\phi}, \boldsymbol{\psi})\right)-b\left((\mathbf{u}, \mathbf{v}), \Pi_{h}(\boldsymbol{\phi}, \boldsymbol{\psi})\right)=\lambda \rho_{\mathrm{F}} c^{2} \int_{\Gamma_{\mathrm{I}}} \operatorname{div} \mathbf{u}\left(\boldsymbol{\phi}_{h} \cdot \boldsymbol{\nu}-\boldsymbol{\psi}_{h} \cdot \boldsymbol{\nu}\right) .
$$

For $\left(\boldsymbol{\phi}_{h}, \boldsymbol{\psi}_{h}\right) \in \mathbf{V}_{h}$, we have $\boldsymbol{\phi}_{h} \cdot \boldsymbol{\nu}=P_{h}\left(\boldsymbol{\psi}_{h} \cdot \boldsymbol{\nu}\right)$, where $P_{h}$ is the $L^{2}\left(\Gamma_{\mathrm{I}}\right)$ projection onto the piecewise constant functions on $\Gamma_{\mathrm{I}}$. Therefore,

$$
\begin{aligned}
& \left|\int_{\Gamma_{\mathrm{I}}} \operatorname{div} \mathbf{u}\left(\boldsymbol{\phi}_{h} \cdot \boldsymbol{\nu}-\boldsymbol{\psi}_{h} \cdot \boldsymbol{\nu}\right)\right| \\
& \left.\quad=\mid \int_{\Gamma_{\mathrm{I}}}\left[\operatorname{div} \mathbf{u}-P_{h}(\operatorname{div} \mathbf{u})\right]\left[P_{h}\left(\boldsymbol{\psi}_{h} \cdot \boldsymbol{\nu}\right)-\boldsymbol{\psi}_{h} \cdot \boldsymbol{\nu}\right)\right] \mid \\
& \quad \leq\left\|\operatorname{div} \mathbf{u}-P_{h}(\operatorname{div} \mathbf{u})\right\|_{L^{2}\left(\Gamma_{\mathrm{I}}\right)}\left\|P_{h}\left(\boldsymbol{\psi}_{h} \cdot \boldsymbol{\nu}\right)-\boldsymbol{\psi}_{h} \cdot \boldsymbol{\nu}\right\|_{L^{2}\left(\Gamma_{\mathrm{I}}\right)} .
\end{aligned}
$$

Because of Theorem 3.1, $\operatorname{div} \mathbf{u} \in H^{1+\alpha}\left(\Omega_{\mathrm{F}}\right)$ for $\alpha>1 / 2$ and $\|\operatorname{div} \mathbf{u}\|_{H^{1+\alpha}\left(\Omega_{\mathrm{F}}\right)} \leq$ $C\|(\mathbf{u}, \mathbf{v})\|$; hence,

$$
\begin{aligned}
\left\|\operatorname{div} \mathbf{u}-P_{h}(\operatorname{div} \mathbf{u})\right\|_{L^{2}\left(\Gamma_{\mathrm{I}}\right)} & \leq \boldsymbol{C h}\|\operatorname{div} \mathbf{u}\|_{H^{1}\left(\Gamma_{\mathrm{I}}\right)} \leq \boldsymbol{C h}\|\operatorname{div} \mathbf{u}\|_{H^{3 / 2}\left(\Omega_{\mathrm{F}}\right)} \\
& \leq \boldsymbol{C h}\|(\mathbf{u}, \mathbf{v})\| .
\end{aligned}
$$

On the other hand,

$$
\begin{aligned}
\left\|\boldsymbol{\psi}_{h} \cdot \boldsymbol{\nu}-P_{h}\left(\boldsymbol{\psi}_{h} \cdot \boldsymbol{\nu}\right)\right\|_{L^{2}\left(\Gamma_{\mathrm{I}}\right)} \leq & \left\|\left(I-P_{h}\right)(\boldsymbol{\psi} \cdot \boldsymbol{\nu})\right\|_{L^{2}\left(\Gamma_{\mathrm{I}}\right)} \\
& +\left\|\left(I-P_{h}\right)\left(\boldsymbol{\psi} \cdot \boldsymbol{\nu}-\boldsymbol{\psi}_{h} \cdot \boldsymbol{\nu}\right)\right\|_{L^{2}\left(\Gamma_{\mathrm{I}}\right)} .
\end{aligned}
$$

Since $(\boldsymbol{\phi}, \boldsymbol{\psi}) \in E(\mathbf{X})$, by applying Theorem 3.1 once more, we have $\boldsymbol{\psi} \in\left[H^{1+\beta}\left(\Omega_{\mathrm{S}}\right)\right]^{2}$ with $\beta \in(0,1]$ and $\|\boldsymbol{\psi}\|_{\left[H^{1+\beta}\left(\Omega_{\mathrm{S}}\right)\right]^{2}} \leq \boldsymbol{C}\|(\boldsymbol{\phi}, \boldsymbol{\psi})\|$. So, for $r:=\min \{1, \beta+1 / 2\}$, we have for the first term on the right of (3.3)

$$
\begin{aligned}
\left\|\left(I-P_{h}\right)(\boldsymbol{\psi} \cdot \boldsymbol{\nu})\right\|_{L^{2}\left(\Gamma_{\mathrm{I}}\right)} & \leq \boldsymbol{C} h^{r}\|\boldsymbol{\psi}\|_{\left[H^{1+\beta}\left(\Omega_{\mathrm{S}}\right)\right]^{2}} \\
& \leq \boldsymbol{C} h^{r}\|(\boldsymbol{\phi}, \boldsymbol{\psi})\| .
\end{aligned}
$$

For the second term we have

$$
\begin{aligned}
& \|(I-\left.P_{h}\right)\left(\boldsymbol{\psi} \cdot \boldsymbol{\nu}-\boldsymbol{\psi}_{h} \cdot \boldsymbol{\nu}\right) \|_{L^{2}\left(\Gamma_{\mathrm{I}}\right)} \\
&\left.\quad \leq\left\|\boldsymbol{\psi} \cdot \boldsymbol{\nu}-\boldsymbol{\psi}_{h} \cdot \boldsymbol{\nu}\right\|_{L^{2}\left(\Gamma_{\mathrm{I}}\right)} \leq \boldsymbol{C}\left\|\boldsymbol{\psi}-\boldsymbol{\psi}_{h}\right\|_{\left[H^{1}\left(\Omega_{\mathrm{S}}\right)\right.}\right]^{2} \\
& \quad \leq \boldsymbol{C} h^{\gamma}\|(\boldsymbol{\phi}, \boldsymbol{\psi})\| ;
\end{aligned}
$$

in the last inequality we have used that $\left\|(\boldsymbol{\phi}, \boldsymbol{\psi})-\Pi_{h}((\boldsymbol{\phi}, \boldsymbol{\psi}))\right\| \leq \boldsymbol{C} \gamma_{h}\|(\boldsymbol{\phi}, \boldsymbol{\psi})\|=$ $\boldsymbol{C} h^{\gamma}\|(\phi, \psi)\|$ because of the particular form of property $\mathbf{P 2}$ valid for this problem. 
Now, since $\gamma=\min \{\alpha, \beta\} \leq r$, we have that (3.3), (3.4) and (3.5) yield

$$
\left\|\boldsymbol{\psi}_{h} \cdot \boldsymbol{\nu}-P_{h}\left(\boldsymbol{\psi}_{h} \cdot \boldsymbol{\nu}\right)\right\|_{L^{2}\left(\Gamma_{\mathrm{I}}\right)} \leq \boldsymbol{C} h^{\gamma}\|(\boldsymbol{\phi}, \boldsymbol{\psi})\|,
$$

which together with (3.1) and (3.2) shows that

$$
\left|\int_{\Gamma_{\mathrm{I}}} \operatorname{div} \mathbf{u}\left(\boldsymbol{\phi}_{h} \cdot \boldsymbol{\nu}-\boldsymbol{\psi}_{h} \cdot \boldsymbol{\nu}\right)\right| \leq \boldsymbol{C} h^{1+\gamma}\|(\mathbf{u}, \mathbf{v})\|\|(\boldsymbol{\phi}, \boldsymbol{\psi})\|,
$$

proving the lemma.

Now, we conclude the main theorem of this section.

Theorem 3.5. There exist positive constants $\boldsymbol{C}$ and $h_{0}$ such that, for $h \leq h_{0}$,

$$
\max _{i=1, \ldots, m}\left|\lambda-\lambda_{i h}\right| \leq \boldsymbol{C} h^{2 \gamma}
$$

with $\gamma:=\min \{\alpha, \beta\}$ and $\alpha$ and $\beta$ as in Theorem 3.1.

Proof. This is a direct application of Theorem 2.3, Lemma 3.1 and the properties $\mathbf{P 1}$ and $\mathbf{P 2}$ valid for this problem.

Theorem 3.5 shows that the order of convergence for the eigenvalues doubles that for the eigenfunctions. Numerical evidence of this improved order had been reported in [5]; however, to the authors' knowledge, it has not been proved before. Furthermore, since ours is a nonconforming approximation of a noncompact operator, neither the theory in [10] nor that in [13] covers this case.

\section{THE INCOMPRESSIBLE CASE}

In [3], it has been proved that the method in [2] described in the previous section can be adapted to the case of an incompressible fluid. A problem with such a fluid can be thought of as a limit case of that with a compressible one as the acoustic speed $c$ goes to $\infty$.

For a perfectly incompressible fluid, the divergence of its displacements vanishes and hence it is convenient to consider the pressure of the fluid as a new variable. In this way we obtain a variational problem for the vibration modes of an elastic vessel completely filled with the fluid (as in Figure 1):

$\mathbf{V P}_{\mathrm{I}}$ : Find $\lambda \in \mathbf{R}$ and $(\mathbf{u}, \mathbf{v}, p) \in \mathbf{V} \times L^{2}\left(\Omega_{\mathrm{F}}\right),(\mathbf{u}, \mathbf{v}, p) \neq(\mathbf{0}, \mathbf{0}, 0)$, such that

$$
\begin{aligned}
a((\mathbf{u}, \mathbf{v}),(\boldsymbol{\phi}, \boldsymbol{\psi})) & -\int_{\Omega_{\mathrm{F}}} p \operatorname{div} \boldsymbol{\phi}=\lambda b((\mathbf{u}, \mathbf{v}),(\boldsymbol{\phi}, \boldsymbol{\psi})), \quad \forall(\boldsymbol{\phi}, \boldsymbol{\psi}) \in \mathbf{V}, \\
& -\int_{\Omega_{\mathrm{F}}} q \operatorname{div} \mathbf{u}=0, \quad \forall q \in L^{2}\left(\Omega_{\mathrm{F}}\right),
\end{aligned}
$$

with $p$ being the pressure into the fluid and with the same notation as in $\S 3$, except for the bilinear form $a$ which, in this case, is defined by

$$
a((\mathbf{u}, \mathbf{v}),(\phi, \psi)):=\int_{\Omega_{\mathrm{S}}} \boldsymbol{\sigma}(\mathbf{v}): \varepsilon(\boldsymbol{\psi}), \quad(\mathbf{u}, \mathbf{v}),(\phi, \psi) \in \mathbf{X} .
$$

In [3], $\mathbf{V P}_{\mathrm{I}}$ is shown to be a well-posed mixed problem in the sense that the bilinear forms involved satisfy the standard Brezzi conditions (see, for instance, [7]). Proceeding as in [3], we can eliminate the pressure $p$ in $\mathbf{V} \mathbf{P}_{\mathrm{I}}$, and the following equivalent eigenvalue problem is obtained: 
IP: Find $\lambda \in \mathbf{R}$ and $(\mathbf{u}, \mathbf{v}) \in \mathbf{W},(\mathbf{u}, \mathbf{v}) \neq(\mathbf{0}, \mathbf{0})$, such that

$$
a((\mathbf{u}, \mathbf{v}),(\boldsymbol{\phi}, \boldsymbol{\psi}))=\lambda b((\mathbf{u}, \mathbf{v}),(\boldsymbol{\phi}, \boldsymbol{\psi})), \quad \forall(\boldsymbol{\phi}, \boldsymbol{\psi}) \in \mathbf{W},
$$

where $\mathbf{W}:=\left\{(\mathbf{u}, \mathbf{v}) \in \mathbf{V}: \operatorname{div} \mathbf{u}=0\right.$ in $\left.\Omega_{\mathrm{F}}\right\}$.

Let $A: \mathbf{X} \longrightarrow \mathbf{X}$ be defined by $A(\mathbf{u}, \mathbf{v}) \in \mathbf{W}$ and

$$
a(A(\mathbf{u}, \mathbf{v}),(\boldsymbol{\phi}, \boldsymbol{\psi}))=b((\mathbf{u}, \mathbf{v}),(\boldsymbol{\phi}, \boldsymbol{\psi})), \quad \forall(\boldsymbol{\phi}, \boldsymbol{\psi}) \in \mathbf{W} .
$$

All the results of the previous section regarding the spectrum and eigenfunctions of $A$ remain valid in this case, with $\mathbf{V}$ substituted by $\mathbf{W}$ and $\mathbf{V P}$ by IP. In particular, the analogue of Theorem 3.1 is the following (see [3]):

Theorem 4.1. Let $(\mathbf{u}, \mathbf{v})$ be an eigenfunction of $A$ with corresponding eigenvalue $\lambda \in(0,1)$. Let $p \in L^{2}\left(\Omega_{\mathrm{F}}\right)$ be such that $\left(\frac{1}{\lambda},(\mathbf{u}, \mathbf{v}, p)\right)$ is a solution of $\mathbf{V} \mathbf{P}_{\mathrm{I}}$. Then $\mathbf{u} \in\left[H^{\alpha}\left(\Omega_{\mathrm{F}}\right)\right]^{2}, \mathbf{v} \in\left[H^{1+\beta}\left(\Omega_{\mathrm{S}}\right)\right]^{2}, p \in H^{1+\alpha}\left(\Omega_{\mathrm{F}}\right)$ and

$$
\left.\|\mathbf{u}\|_{\left[H^{\alpha}\left(\Omega_{\mathrm{F}}\right)\right.}\right]^{2}+\|\mathbf{v}\|_{\left[H^{1+\beta}\left(\Omega_{\mathrm{S}}\right)\right]^{2}}+\|p\|_{H^{1+\alpha}\left(\Omega_{\mathrm{F}}\right)} \leq \boldsymbol{C}\|(\mathbf{u}, \mathbf{v})\|,
$$

with $\alpha \in(1 / 2,1]$ and $\beta \in(0,1]$ and with $\boldsymbol{C}$ a positive constant.

The finite element spaces introduced in $\S 3$ are also useful in the incompressible case. Furthermore, let $\mathbf{W}_{h}:=\left\{(\mathbf{u}, \mathbf{v}) \in \mathbf{V}_{h}: \operatorname{div} \mathbf{u}=0\right.$ in $\left.\Omega_{\mathrm{F}}\right\}$. Let $A_{h}: \mathbf{X} \longrightarrow \mathbf{X}$ be defined by $A_{h}(\mathbf{u}, \mathbf{v}) \in \mathbf{W}_{h}$ and

$$
a\left(A_{h}(\mathbf{u}, \mathbf{v}),(\boldsymbol{\phi}, \boldsymbol{\psi})\right)=b((\mathbf{u}, \mathbf{v}),(\boldsymbol{\phi}, \boldsymbol{\psi})), \quad \forall(\boldsymbol{\phi}, \boldsymbol{\psi}) \in \mathbf{W}_{h} .
$$

In [3] it is shown that the spectrum and the eigenfunctions of $A_{h}$ approximate those of $A$; implementation issues and numerical experiments are reported in [4]. Notice that, in particular, the restriction $\operatorname{div} \mathbf{u}=0$ in the definition of $\mathbf{W}_{h}$ is easy to deal with, since $\mathbf{W}_{h}=\left\{(\operatorname{curl} \xi, \mathbf{v}) \in \mathbf{V}_{h}: \xi \in L_{h}\left(\Omega_{\mathrm{F}}\right)\right\}$.

Properties $\mathbf{P 1}$ and $\mathbf{P 2}$ as in the previous section are shown to remain valid in the incompressible case in [3]; these properties are used therein to show the analogues of Theorem 3.2 (with $\mathbf{K} \cap \mathbf{W}_{h}$ instead of $\mathbf{K} \cap \mathbf{V}_{h}$ ) and Theorem 3.3.

Given $\lambda \in(0,1)$, an eigenvalue of $A$ of multiplicity $m$, for $h$ small enough there exist $m$ eigenvalues of $A_{h}, \lambda_{1 h}, \ldots, \lambda_{m h}$, (repeated according to their multiplicities) converging to $\lambda$. Let $E(\mathbf{X})$ and $F_{h}(\mathbf{X})$ denote the respective eigenspaces. Theorem 3.4 is also valid in the incompressible case (see [3]).

As a consequence, $\mathbf{P} 2$ is also valid with $\mathbf{V}_{h}$ substituted by $\mathbf{W}_{h}$. Property $\mathbf{P} \mathbf{1}$ is obviously valid for $\mathbf{W}_{h} \subset \mathbf{V}_{h}$. Lemma 3.1 is valid too in the incompressible case; in fact, its proof reduces to that of the compressible case by substituting div $\mathbf{u}$ by $p$ and by using Theorem 4.1 to bound $\|p\|_{H^{3 / 2}\left(\Omega_{\mathrm{F}}\right)}$ in the analogue of (3.2).

Therefore, we may conclude, also in the incompressible case, that there is a double order of convergence for the eigenvalues:

Theorem 4.2. There exist positive constants $C$ and $h_{0}$ such that, for $h \leq h_{0}$,

$$
\max _{i=1, \ldots, m}\left|\lambda-\lambda_{i h}\right| \leq \boldsymbol{C} h^{2 \gamma}
$$

with $\gamma:=\min \{\alpha, \beta\}$ and $\alpha$ and $\beta$ as in Theorem 4.1 . 


\section{REFERENCES}

1. I. Babuška and J. Osborn, Eigenvalue problems, in Handbook of Numerical Analysis, Vol. II (ed. P. G. Ciarlet and J. L. Lions) North Holland, Amsterdam, 1991. CMP 91:14

2. A. Bermúdez, R. Durán, M.A. Muschietti, R. Rodríguez and J. Solomin, Finite element vibration analysis of fluid-solid systems without spurious modes, SIAM J. Numer. Anal., 32 (1995), 1280-1295. CMP 95:15

3. A. Bermúdez, R. Durán and R. Rodríguez, Finite element analysis of compressible and incompressible fluid-solid systems, Math. Comp. (submitted)

4. A. Bermúdez, R. Durán and R. Rodríguez, Finite element solution of incompressible fluidstructure vibration problems, Internat. J. Numer. Methods Engrg. (submitted)

5. A. Bermúdez and R. Rodríguez, Finite element computation of the vibration modes of a fluid-solid system, Comp. Methods in Appl. Mech. and Eng., 119 (1994) pp. 355-370. MR 95j:73064

6. J. Boujot, Mathematical formulation of fluid-structure interaction problems, $\mathrm{M}^{2} \mathrm{AN}, \mathbf{2 1}$ (1987) 239-260. MR 88j:73032

7. F. Brezzi, M. Fortin, Mixed and Hybrid Finite Element Methods, Springer-Verlag, New York, 1991. MR 92d:65187

8. H. C. Chen and R. L. Taylor, Vibration analysis of fluid-solid systems using a finite element displacement formulation, Internat. J. Numer. Meth. Eng., 29 (1990) 683-698.

9. J. Descloux, N. Nassif and J. Rappaz, On spectral approximation. Part I: The problem of convergence., R.A.I.R.O. Anal. Numér., 12 (1978) 97-112. MR 58:3404a

10. J. Descloux, N. Nassif and J. Rappaz, On spectral approximation. Part 2: Error estimates for the Galerkin methods, R.A.I.R.O. Anal. Numér., 12 (1978) 113-119. MR 58:34046

11. M. Hamdi, Y. Ouset and G. Verchery, A displacement method for the analysis of vibrations of coupled fluid-structure systems, Internat. J. Numer. Methods Eng., 13 (1978) 139-150.

12. H.J-P. Morand and R. Ohayon, Interactions fluides-structures, Recherches en Mathématiques Appliquées, 23, Masson, Paris, 1992. MR 93m:73031

13. B. Mercier, J. Osborn, J. Rappaz and P. A. Raviart, Eigenvalue approximation by mixed and hybrid methods, Math. Comp., 36 (1981) 427-453. MR 82b:65108

14. L. Olson and K. Bathe, Analysis of fluid-structure interactions. A direct symmetric coupled formulation based on the fluid velocity potential, Comp. Struct., 21 (1985) 21-32.

15. P. A. Raviart and J. M. Thomas, A mixed finite element method for second order elliptic problems, Mathematical Aspects of Finite Element Methods. Lecture Notes in Mathematics, 606, Springer-Verlag, Berlin, Heidelberg, New York, 1977, 292-315. MR 58:3547

16. J. H. Wilkinson, The algebraic eigenvalue problem, Clarendon Press, Oxford, 1965.

Departamento de Ingeniería Matemática, Universidad de Concepción, Casilla 4009, Concepción, Chile

E-mail address: rodolfo@gauss.cfm.udec.cl

Departamento de Matemática, Facultad de Ciencias Exactas, Universidad Nacional de La Plata, C.C. 172, 1900 La Plata, Argentina

E-mail address: solo@mate.unlp.edu.ar 